\title{
Plasmablasts as a biomarker for lgG4-related disease, independent of serum IgG4 concentrations
}

\author{
Zachary S Wallace, ${ }^{1}$ Hamid Mattoo, ${ }^{2}$ Mollie Carruthers, ${ }^{1}$ Vinay S Mahajan, ${ }^{2}$ \\ Emanuel Della Torre, ${ }^{2}$ Hang Lee, ${ }^{3}$ Maria Kulikova, ${ }^{2}$ Vikram Deshpande, ${ }^{4}$ Shiv Pillai, ${ }^{2}$ \\ John H Stone ${ }^{1}$
}

\begin{abstract}
Handling editor Tore K Kvien
- Additional material is published online only. To view please visit the journal online (http://dx.doi.org/10.1136/ annrheumdis-2014-205233).

${ }^{1}$ Division of Rheumatology, Allergy, and Immunology, Massachusetts General Hospital, Boston, Massachusetts, USA

${ }^{2}$ Massachusetts General Hospital Cancer Center, Boston, Massachusetts, USA

${ }^{3}$ Biostatistics Center,

Massachusetts General Hospital, Boston,

Massachusetts, USA

${ }^{4}$ Department of Pathology, Massachusetts General

Hospital, Boston,

Massachusetts, USA
\end{abstract}

\section{Correspondence to}

Dr John H Stone,

Rheumatology Clinic/Yawkey 2,

Massachusetts General

Hospital, 55 Fruit Street,

Boston, MA 02114, USA:

jhstone@partners.org

Received 12 January 2014

Revised 7 April 2014

Accepted 16 April 2014

Published Online First

9 May 2014

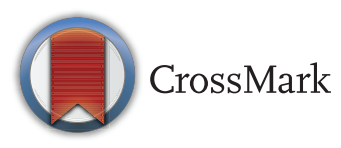

\section{SLinked}

- http://dx.doi.org/10.1136/ annrheumdis-2013-204907

- http://dx.doi.org/10.1136/

annrheumdis-2014-205476

To cite: Wallace $\mathrm{ZS}$,

Mattoo H, Carruthers M,

et al. Ann Rheum Dis

2015;74:190-195.

\section{ABSTRACT}

Objectives We examined the utility of circulating total and lgG4+ plasmablasts as biomarkers of diagnosis and disease activity in IgG4-related disease (IgG4-RD).

Materials methods We evaluated patients with active, untreated, biopsy-proven IgG4-RD affecting various organs. Flow cytometry was used to measure total plasmablast and IgG4+ plasmablast counts by gating peripheral blood for CD19lowCD38+CD20-CD27 + cells and CD19lowCD38+CD20-CD27+lgG4+ cells. Serum IgG4 concentrations were measured by nephelometry. We compared 37 lgG4-RD patients to 35 controls, including healthy individuals $(n=14)$ and patients with other inflammatory diseases before treatment $(n=21)$.

Results The lgG4-RD patients' mean age was 59, and $68 \%$ were male. Fourteen patients (38\%) had three or more organs involved. The IgG4-RD patients had substantially elevated total plasmablast counts (median $4698 / \mathrm{mL}$, range $610-79524 / \mathrm{mL}$ ) compared to both untreated disease controls (median 592/mL, range 19$4294 / \mathrm{mL} ; \mathrm{p}<0.001$ ) and healthy controls (median $94 / \mathrm{mL}$, range $1-653 / \mathrm{mL} ; p<0.001)$. Thirteen IgG4-RD patients $(36 \%)$ had normal serum IgG4 concentrations (mean $60 \mathrm{mg} / \mathrm{dL}$, range 5-123 mg/dL, normal <135 mg/dL). However, the median plasmablast count was not significantly lower in that subset with normal serum lgG4 concentrations $(3784 / \mathrm{mL})$ compared to those with elevated serum IgG4 (5155/mL) $(p=0.242)$. Among the 12 rituximab (RTX)-treated patients, the median plasmablast level during disease flare was $6356 / \mathrm{mL}$ (range 1123$41589 / \mathrm{mL}$ ), declining to $1419 / \mathrm{mL}$ (range $386 / \mathrm{mL}-4150$ / $\mathrm{mL})$ during remission $(p<0.01)$.

Conclusions Circulating plasmablasts are elevated in active lgG4-RD, even in patients with normal serum lgG4 concentrations. Plasmablast counts are a potentially useful biomarker for diagnosis, assessing response to treatment, and determining the appropriate time for re-treatment.

\section{INTRODUCTION}

IgG4-related disease (IgG4-RD) is an immunemediated, fibro-inflammatory disease that leads to tissue damage, organ dysfunction and, if untreated, organ failure. ${ }^{1}$ The disease can affect almost any anatomic location, but the sites involved most commonly are the pancreas, salivary glands, orbital adnexa, lymph nodes and retroperitoneum. ${ }^{2} 3$ IgG4-RD, typically diagnosed in middle-aged individuals, is characterised by a male predominance except with regard to organs of the head and neck (eg, the salivary glands and orbits), where the gender distribution is approximately equal. ${ }^{3-5}$ The epidemiology of IgG4-RD remains poorly understood because of its recognition only recently as a multi-organ disease. However, IgG4-RD accounts for many conditions once regarded as disparate, single-organ disorders. ${ }^{6}$

The current gold standard for the diagnosis of IgG4-RD is the identification of characteristic histology and immunohistochemistry features through biopsy. These pathology features are consistent across the full range of organs affected by IgG4-RD. ${ }^{7-9}$ However, histopathological variation can occur according to the stage of the lesion in that longstanding disease may be predominately fibrotic and acellular. Confirming the diagnosis of IgG4-RD in such cases can be difficult. ${ }^{10}$ Moreover, IgG4-RD organ pathology and IgG4-RD mimickers, such as granulomatosis with polyangiitis (GPA) (formerly Wegener's), sarcoidosis, histiocytoses and malignancies (eg, lymphoma and adenocarcinoma of the pancreas) may share similar features, including an IgG4-positive plasma cell infiltrate. ${ }^{11}{ }^{12}$ Reliance upon serum IgG4 concentrations to diagnose IgG4-RD is similarly problematic because both the specificity and positive predictive value of serum IgG4 concentrations are poor. ${ }^{13} 14$

B cell depletion appears to be an effective treatment strategy for IgG4-RD, but the assessment of disease activity following treatment and appropriate timing of re-treatment remains challenging. In patients with a normal serum IgG4 concentration at baseline, for example, serial measurements are of limited to no utility. Even in patients with elevated baseline IgG4 concentrations, the decline in IgG4 following successful treatment is variable, and measurements frequently remain elevated, albeit lower. A biomarker with high sensitivity and specificity for active IgG4-RD would be of tremendous value for confirming diagnostic suspicion, assessing response to treatment, and identifying impending relapse.

Both humoral immunity and cell-mediated immunity have been implicated in the pathophysiology of IgG4-RD. ${ }^{15-17}$ The disease's response to depletion of peripheral CD20+ naive and memory $\mathrm{B}$ cells with rituximab (RTX) treatment supports the hypothesis that B cells play an important role in IgG4-RD pathogenesis, either directly or through the effects of $\mathrm{B}$ cell depletion on $\mathrm{T}$ cell behaviour, as is theorised in other conditions. ${ }^{18-20}$ 
Plasmablasts, derived from the $\mathrm{B}$ cell lineage and characterised as CD19lowCD20-CD38+CD27+, comprise a stage intermediate between activated $\mathrm{B}$ cells and plasma cells. ${ }^{21}$ Plasmablasts are generally rare in the peripheral blood of healthy individuals, ${ }^{21} 22$ but expansions are observed briefly during responses to infection or vaccination. ${ }^{23}$ In contrast, in the setting of autoimmunity and persistent self-antigen(s), plasmablasts can circulate for prolonged periods. ${ }^{21} 2425$

Circulating plasmablasts have been described previously in inflammatory bowel disease, rheumatoid arthritis (RA), systemic lupus erythematosus and multiple myeloma. ${ }^{21} 22$ 24-27 One case report describes a patient with IgG4-RD who had elevated circulating plasmablasts that declined with corticosteroid treatment. ${ }^{28}$ In this study, we describe the clinical characteristics of patients with active, untreated IgG4-RD and elevated plasmablast levels in blood, stratified according to serum IgG4 concentration. We also report the relationship between total and IgG4+ plasmablasts and disease activity, and describe serial assessments of plasmablasts following treatment.

\section{MATERIALS AND METHODS}

\section{Patients}

This study was approved by the institutional review board and all subjects provided informed, written consent. From the

Table 1 Clinical and laboratory characteristics of IgG4-RD patients

\begin{tabular}{|c|c|c|}
\hline & $\begin{array}{l}\text { All IgG4-RD } \\
\text { patients }(n=37)\end{array}$ & $\begin{array}{l}\text { IgG4-RD } \\
\text { patients } \\
\text { treated with } \\
\text { RTX }(n=12)\end{array}$ \\
\hline Age, mean (years) & 59 (range 33-82) & $\begin{array}{l}60.4 \\
\text { (range 44-77) }\end{array}$ \\
\hline Gender ( $\%$ male) & $68 \%$ & $82 \%$ \\
\hline Race & $76 \%$ Caucasian & $64 \%$ Caucasian \\
\hline Plasmablast count, median (/mL) & $\begin{array}{l}4698 \\
\text { (range 610-79 524) }\end{array}$ & $\begin{array}{l}6356 \text { (range } \\
1145-41589 \text { ) }\end{array}$ \\
\hline $\begin{array}{l}\text { Serum IgG4, mean } \\
\text { (normal }<135 \mathrm{mg} / \mathrm{dL} \text { ) }\end{array}$ & 612 (range 5.3-4780) & $\begin{array}{l}1014 \\
\text { (range 26-4780) }\end{array}$ \\
\hline Number with normal serum IgG4 (\%) & $13(36 \%)$ & $3(25 \%)$ \\
\hline C3, mean (normal 86-184 mg/dL) & 94.3 & 90 \\
\hline C4, mean (normal $16-38 \mathrm{mg} / \mathrm{dL}$ ) & 17.4 & 14.4 \\
\hline ESR, mean (normal 0-20 mm/h) & 37 & 39.4 \\
\hline CRP, mean (normal $<8 \mathrm{mg} / \mathrm{L}$ ) & 16.6 & 15.4 \\
\hline IgG4-RD Responder Index (mean) & 11 (range 3-36) & $\begin{array}{l}13.8 \\
\text { (range 3-36) }\end{array}$ \\
\hline Average no. of organs involved & 2.6 (range 1-6) & 2.8 (range 1-6) \\
\hline Orbit & 7 patients & \\
\hline Parotid & 7 patients & \\
\hline Sinusitis & 1 patient & \\
\hline Palate & 3 patients & \\
\hline Lymphadenopathy & 6 patients & \\
\hline Lungs & 6 patients & \\
\hline RPF & 6 patients & \\
\hline Sclerosing mesenteritis & 1 patient & \\
\hline Pancreas & 8 patients & \\
\hline Liver & 2 patients & \\
\hline Biliary tract & 2 patients & \\
\hline Gallbladder & 1 patient & \\
\hline Kidney & 3 patients & \\
\hline Other & 1 patient & \\
\hline
\end{tabular}

CRP, C-reactive protein; ESR, erythrocyte sedimentation rate; IgG4-RD, IgG4-related disease; RPF, retroperitoneal fibrosis; RTX, rituximab. database of the Massachusetts General Hospital Center for IgG4-Related Disease, we identified 37 sequentially evaluated patients who had active, untreated disease. The IgG4-RD patients were compared to 14 healthy controls and to 21 disease controls with active, untreated inflammatory diseases or malignancies at the time their blood samples were drawn. The 21 disease control subjects included five patients with RA, four with GPA, two with pancreatic cancer, one with diffuse large B cell lymphoma, three with sarcoidosis, and one each with livedoid vasculopathy, Sjögren's syndrome, primary biliary cirrhosis, chronic pain, acute Lyme disease, and gout. Twelve of the patients with active, untreated IgG4-RD were treated with RTX (two $1000 \mathrm{mg}$ doses separated by 15 days). Peripheral blood was analysed by flow cytometry before and after this therapy.

\section{Pathology}

All 37 patients had clinical histories consistent with IgG4-RD and biopsies of involved organs met criteria for a definite diagnosis. ${ }^{7}$ Haematoxylin and eosin-stained slides were reviewed to determine the histopathological features. Immunohistochemistry for IgG4 and IgG was performed using antibodies to IgG4 (Zymed; 1:200 dilution) and IgG (Dako; 1:3000 dilution). For each case, the number of plasma cells staining for IgG4 was assessed in three non-overlapping high-power fields (magnification $\times 400$ ). ${ }^{7}$ The fields with the highest degree of IgG4 reactivity were counted. The number of IgG4+ plasma cells was then divided by the total number of IgG + plasma cells in these fields to determine the $\operatorname{IgG} 4+: \operatorname{IgG}+$ plasma cell ratio.

\section{Disease activity}

The IgG4-RD Responder Index (RI) was used to identify patients with active disease, defined by an RI score $\geq 3 .{ }^{29}$ In serial assessments, disease activity was categorised as 'flare' or 'not flare'. ${ }^{30}$ Details regarding demographic features and reported standard laboratory tests were collected from the electronic medical record; one patient did not have data on serum IgG4 concentrations at baseline available. Patients with other immune-mediated conditions were determined to have active disease based on the assessments of their treating providers. None of the controls had received treatment for their condition prior to blood sampling.

\section{Flow cytometry}

Flow cytometry was used to measure the absolute plasmablast count per millilitre by gating peripheral blood for CD19lowCD20 $-\mathrm{CD} 38+\mathrm{CD} 27+$ and staining for IgG4 + cells in a subset of patients. Flow cytometry was performed on all samples at the time of patients' presentations with active, untreated disease. Patients who were treated with RTX were re-evaluated by flow cytometry within 3-6 months. Serum IgG4 concentrations were measured by immunonephelometry using a Siemens BNII instrument and reagent sets obtained from either Siemens or The Binding Site. The prozone effect, known to occur with some nephelometry assays in the setting of high antigen excess, was avoided by diluting the samples sufficiently until consistent measurements were obtained from one serial dilution to the next. ${ }^{31}$

\section{Statistical analysis}

All statistical tests were performed using SPSS V.21. Statistical differences were found by paired and unpaired Student $t$ tests and by non-parametric tests, including all assessments of plasmablast levels. Linear correlations were measured by Pearson's correlation coefficient. Receiver operating characteristic (ROC) curves were generated based on the assumption that ascending 
rank of plasmablast counts would increase the likelihood of an IgG4-RD diagnosis. A $p$ value of $<0.05$ was considered significant for all statistical testing.

\section{RESULTS}

\section{Clinical characteristics and demographics}

The clinical characteristics of the 37 IgG4-RD patients and the subset of 12 who were treated with RTX are described in table 1. The mean age of the IgG4-RD group was 59 years (range 33-82). Twenty-five $(68 \%)$ of the patients were male. Fourteen $(38 \%)$ patients had at least three organs involved (range 3-6), while the remaining 23 had active IgG4-RD in only one or two organs. Of the 12 patients treated with RTX, four experienced disease flares an average of 8 months after the initial infusion.

The most commonly involved organs were the lymph nodes and submandibular glands (11 cases each). Lymphadenopathy was the sole disease manifestation in only one patient. Other frequent sites of involvement included the pancreas (8 cases), parotid gland ( 7 cases) and orbit ( 7 cases). In the 37 IgG4-RD patients overall, 18 different anatomical locations were involved in at least one patient. The average IgG4-RD RI score was 11 (range 3-36). The mean ages of the disease controls and healthy controls were 53 years (range 25-81) and 43 years (range 23-60), respectively. Twenty-nine percent of the disease controls and $60 \%$ of the healthy controls were males.

\section{Plasmablast counts}

All IgG4-RD patients had expanded circulating plasmablast populations (median $4698 / \mathrm{mL}$, range $610-79524 / \mathrm{mL}$ ). The median plasmablast count among healthy controls was $94 / \mathrm{mL}$ (range 1-653/mL; p $<0.001$ compared with IgG4-RD) (figure 1), while that of patients with other immune-mediated conditions and malignancies was 592.0/mL (range 19-4294/mL; p $<0.001$ compared with IgG4-RD) (figure 1). Several patients with other immune-mediated conditions and one healthy control had elevated plasmablast counts. These values were $644 / \mathrm{mL}$ (a patient with GPA), 653/mL (healthy control), 752/mL (RA), 798/mL (sarcoidosis), 1917/mL (primary biliary cirrhosis), 2428/mL (GPA), 3802/mL (Sjögren's syndrome) and 4294/mL (RA). Male and female IgG4-RD patients had similar plasmablast counts (male median 4093/mL, range 610-79 524/mL; female median $5155 / \mathrm{mL}$, range $910-15528 / \mathrm{mL} ; \mathrm{p}=0.67)$.

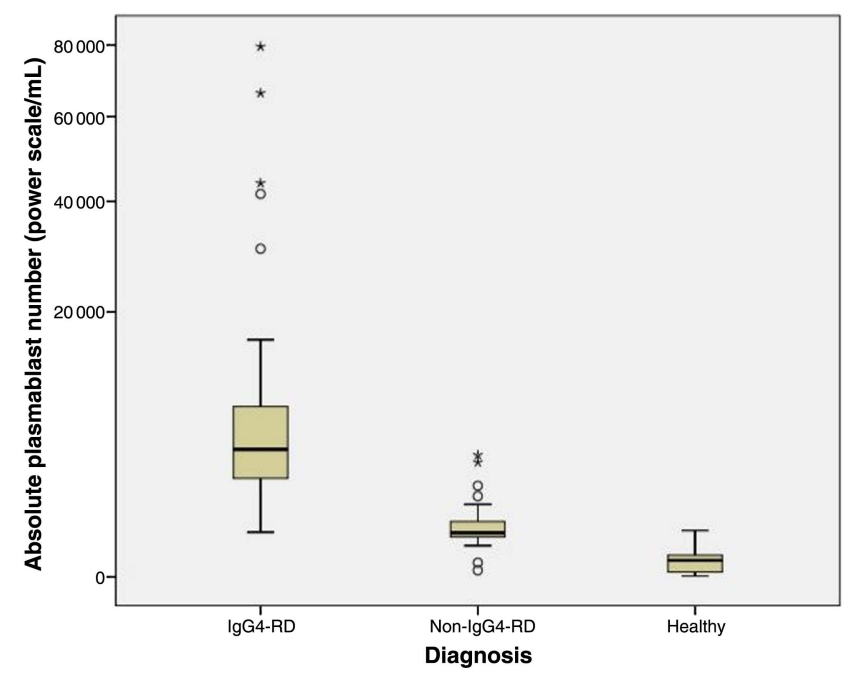

Figure 1 Box plot of median plasmablast count (power scale $/ \mathrm{mL}$ ) by diagnosis. Open circles represent outliers and asterisks represent extreme outliers.

\section{Serum IgG4 concentrations}

Among IgG4-RD patients, 13 (36\%) had normal serum IgG4 concentrations (mean $59.5 \mathrm{mg} / \mathrm{dL}$, range $5.3-123 \mathrm{mg} /$ $\mathrm{dL}$, normal $<135 \mathrm{mg} / \mathrm{dL}$ ). The features of patients with elevated serum IgG4 concentrations are compared to those of patients with normal serum IgG4 concentrations in table 2 . Patients with elevated serum IgG4 concentrations (mean $924 \mathrm{mg} / \mathrm{dL}$, range $138-4780 \mathrm{mg} / \mathrm{dL}$, normal $<135 \mathrm{mg} / \mathrm{dL}$ ) were more likely to have involvement of three or more organs $(57 \%$ vs $15 \% ; \mathrm{p}<0.01)$. In addition, IgG4-RD patients with elevated serum IgG4 concentrations had a greater mean number of organs involved than did those with normal serum IgG4 (2.9 vs $1.7 ; \mathrm{p}<0.01)$, and were also more likely to have abnormal inflammatory markers (low C3 and $\mathrm{C} 4$, and high erythrocyte sedimentation rate and C-reactive protein) (table 2). Patients with normal serum IgG4 concentrations had mean RI scores nearly seven points lower compared to those with elevated serum IgG4 concentrations (mean 6.5 (range 3-14) vs 13.3 (range 6-36); $\mathrm{p}<0.01)$. The mean serum IgG4 concentration was higher among male patients (mean $728 \mathrm{mg} / \mathrm{dL}$ vs $378 \mathrm{mg} / \mathrm{dL}$ for females; $\mathrm{p}=0.05$ ).

Table 2 shows data from a comparison among IgG4-RD patients, stratified according to whether their serum IgG4 concentrations were elevated $(>135 \mathrm{mg} / \mathrm{dL})$ or normal.

\section{Plasmablast, IgG4, disease extent and disease activity correlations}

No differences were observed between the plasmablast counts of IgG4-RD patients with elevated as opposed to normal serum IgG4 values (medians $5525 / \mathrm{mL}$ and $3784 / \mathrm{mL} ; \mathrm{p}=0.24$ ). Plasmablast counts were significantly different between those with multi-organ disease and those with $\leq 2$ organs involved (medians $7370 / \mathrm{mL}$ and $3435 / \mathrm{mL}$, respectively; $\mathrm{p}=0.01$ ). Correlation between the baseline plasmablast counts and the baseline IgG4-RD RI score was modest $(\mathrm{R}=0.17$; $\mathrm{p}=0.16)$.

\section{IgG4+ plasmablasts}

IgG4+ plasmablast levels were assessed in 24 patients with active, untreated IgG4-RD. The median IgG4+ plasmablast level was $2808 / \mathrm{mL}$ (range 203-57 012/mL). In this same group

\begin{tabular}{|c|c|c|c|}
\hline Variable & $\begin{array}{l}\text { Elevated serum } \\
\text { IgG4 }(n=23)\end{array}$ & $\begin{array}{l}\text { Normal serum } \\
\text { lgG4 }(n=13)\end{array}$ & $p$ Value \\
\hline Gender ( $\%$ male) & $65 \%$ & $69 \%$ & 0.81 \\
\hline Age (years) & 64 & 50 & 0.001 \\
\hline $\begin{array}{l}\text { Number of organs } \\
\text { (mean) }\end{array}$ & 2.9 & 1.7 & 0.003 \\
\hline $\begin{array}{l}\text { Percentage with } \geq 3 \\
\text { organs involved }\end{array}$ & $57 \%$ & $15 \%$ & $<0.01$ \\
\hline $\begin{array}{l}\text { Median plasmablast } \\
\text { count }(/ \mathrm{mL})\end{array}$ & 5525 & 3784 & 0.82 \\
\hline Serum IgG4 (mg/dL) & 923.6 & 59.5 & 0.001 \\
\hline C3 (mg/dL) & 82 & 113 & 0.005 \\
\hline $\mathrm{C} 4$ (mg/dL) & 14.9 & 22.1 & 0.017 \\
\hline CRP (mg/L) & 23.4 & 3.1 & 0.008 \\
\hline ESR $(\mathrm{mm} / \mathrm{h})$ & 51 & 11 & $<0.001$ \\
\hline
\end{tabular}


of 24 IgG4-RD patients, the median total number of plasmablasts was $4083 / \mathrm{mL}$ (range $610-79524 / \mathrm{mL}$ ). Thus, IgG4+ plasmablasts accounted for a mean percentage of $61 \%$ of the total plasmablast concentrations (range 14-90\%). There appeared to be trends toward a higher median IgG4+ plasmablast level among patients with elevated as opposed to normal serum IgG4 concentrations $(4631 / \mathrm{mL}$ vs $1372 / \mathrm{mL} ; \mathrm{p}=0.10)$. Similarly, the percentage of plasmablasts that were IgG4+ plasmablasts was higher among those patients with elevated serum IgG4, but this comparison fell short of statistical significance $(67 \%$ vs $49 \%$, respectively; $\mathrm{p}=0.15$ ).

\section{Test characteristics of elevated plasmablast concentrations}

An ROC curve was created to analyse the utility of the plasmablast level as a diagnostic test for IgG4-RD. Overall, plasmablast levels demonstrated excellent performance as a test for IgG4-RD (area under the ROC curve (AUC) 0.96; $\mathrm{p}<0.05$; $95 \%$ CI 0.92 to 0.99 ). A plasmablast value of $900 / \mathrm{mL}$ had a sensitivity of $95 \%$, a specificity of $82 \%$, a positive predictive value of $86 \%$, and a negative predictive value of $97 \%$. A plasmablast value of $2000 / \mathrm{mL}$ had a sensitivity of $87 \%$, a specificity of $91 \%$, a positive predictive value of $91 \%$, and a negative predictive value of $87 \%$.

\section{Change in plasmablast counts and serum IgG4 concentrations with treatment}

Twelve of the patients evaluated with active, untreated disease received a course of RTX (two $1000 \mathrm{mg}$ doses separated by 15 days). Flow cytometry was performed at baseline and at least
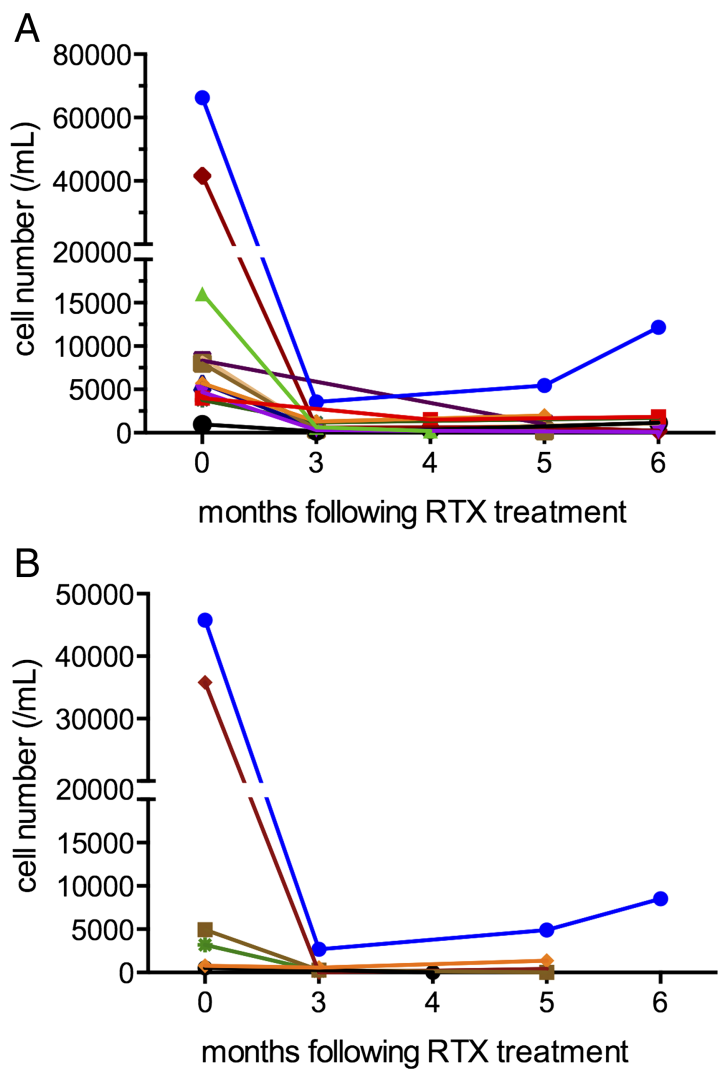

Figure 2 (A) Absolute plasmablast count $(/ \mathrm{mL})$ at baseline (month 0 ) and following rituximab (RTX) infusions. Each line represents a different patient ( $\mathrm{n}=12$ patients). (B) Absolute IgG4+ plasmablast count $(/ \mathrm{mL})$ at baseline (month 0 ) and following RTX infusions. Each line represents a different patient ( $n=6$ patients). The same colour is used for these six patients as in figure 2(A). once between months 3 and 6 after treatment in all patients (figure 2A). The median plasmablast level of $6356 / \mathrm{mL}$ (range $1123-41589 / \mathrm{mL}$ ) during periods of active disease declined to $1419 / \mathrm{mL}$ (range $386-4150 / \mathrm{mL}$ ) during periods of complete or partial remission $(p<0.01$ by related samples Wilcoxon signed rank test) (see online supplement 1 ). In contrast, no significant difference in the mean serum IgG4 concentration was observed between these same periods (mean serum IgG4 concentration with active disease of $1014 \mathrm{mg} / \mathrm{dL}$ (range $26-4780 \mathrm{mg} / \mathrm{dL}$ ) compared with $521 \mathrm{mg} / \mathrm{dL}$ (range $18-2098 \mathrm{mg} / \mathrm{dL}$ ); $\mathrm{p}=0.12$ by paired $t$ test) (see online supplement 2). The mean percentage change in plasmablast level between flare and remission was $66 \%$ (range $-15 \%$ to $98 \%$ ) compared to $23 \%$ (range $-109 \%$ to $75 \%)$ for IgG4 concentrations ( $\mathrm{p}=0.002$ by paired $\mathrm{t}$ test). The mean baseline and follow-up IgG4-RD RI scores were 13.8 during flare (range 3-36) and 4.4 during periods of partial or complete remission (range $0-10 ; \mathrm{p}=0.001$ ) following RTX.

In six patients, IgG4+ and total plasmablast levels were assessed at baseline and 3-6 months following RTX treatment (figure 2B). The median IgG4+ plasmablast level among these patients was $4077 / \mathrm{mL}$ (range 534-45 752/mL) at baseline and $178 / \mathrm{mL}$ (range $0-4906 / \mathrm{mL})$ at $3-6$ months after treatment $(\mathrm{p}=0.046)$.

\section{DISCUSSION}

This study demonstrates that patients with active, untreated IgG4-RD have significant elevations in their circulating plasmablast counts regardless of their serum IgG4 concentrations, and that patients with multi-organ IgG4-RD have higher absolute plasmablast counts than those with involvement of only one or two organs. In addition, plasmablast levels appear to be superior to serum IgG4 concentrations as a biomarker for IgG4-RD. Plasmablast counts decline swiftly following peripheral B cell depletion, and this decline is accompanied by corresponding decreases in disease activity as measured by the IgG4-RD RI. IgG4-RD thus represents an unusual example of an immunemediated condition in which measurement of a single cell type may play a central role in diagnosing, monitoring and managing the disease.

The identification of circulating plasmablast expansion in IgG4-RD has substantial implications for clinical care. The diagnosis of IgG4-RD remains dependent upon biopsy and is therefore subject to the limitations of tissue accessibility (eg, the aorta), the small size of the biopsy specimen (the upper airways, retroperitoneum) and sampling error. Although elevated plasmablast levels can be found in patients with other inflammatory conditions, the expansion seen in patients with IgG4-RD is significantly greater such that levels over $2000 / \mathrm{mL}$ have a high specificity and positive predictive value for this diagnosis. At the same time, blood plasmablast levels of this magnitude maintain a high sensitivity for IgG4-RD. Further, the combination of an extreme elevation in plasmablast count and a substantial elevation in the serum IgG4 concentration suggests a high likelihood of multi-organ disease and should prompt providers to consider more comprehensive evaluations, for example, with additional imaging, as appropriate. Alternatively, a low plasmablast level argues against active IgG4-RD and may suggest another diagnosis.

Measurements of peripheral blood plasmablasts may be particularly useful in patients with normal serum IgG4 concentrations in whom there remains a high clinical suspicion for IgG4-RD and in whom appropriate measures have been undertaken to exclude malignancy. Moreover, the declines observed in circulating plasmablast counts following treatment and the rise before clinical flare in the small number of patients in this series 
who flared during the period of observation, suggest that serial measurements have an important role in following response to treatment, in determining if the intensity of treatment has been sufficient, and in guiding the timing of repeat therapy (data not shown). This final point is particularly critical in patients with organ-threatening disease (eg, IgG4-related sclerosing cholangitis, tubulointerstitial nephritis, aortitis or pachymeningitis) in whom disease recurrence may lead to irreversible damage or organ failure. Other immune-mediated diseases that respond to $\mathrm{B}$ cell depletion, for example, ANCA-associated vasculitis and RA, typically require re-treatment over the course of longitudinal follow-up, but there are no reliable laboratory indicators that predict the timing of disease relapse in advance. Consequently, clinicians are confronted with the need to treat patients at regular intervals-risking overtreatment of some patients-or waiting for clinical disease flares to become evident, placing patients at some risk of irreversible organ damage.

All 37 patients with active, untreated IgG4-RD had elevated plasmablast counts despite the fact that 13 (36\%) had normal serum IgG4 concentrations. This finding is consistent with other studies of serum IgG4 concentrations, which have observed that between $10 \%$ and $30 \%$ of patients have normal serum IgG4 concentrations even in the presence of classic histopathological and immunohistochemical staining features of the disease. ${ }^{14} 32$ Other investigators have demonstrated that serum IgG4 concentrations also have shortcomings as predictors of disease flares. ${ }^{33}$ In our study of patients with active, untreated IgG4-RD, the median plasmablast count did not differ between the two groups with normal and elevated serum IgG4 concentrations, consistent with the concept that the circulating plasmablast count is a more robust diagnostic marker than are serum IgG4 concentrations. Our observation that plasmablast levels differ significantly during periods of disease flare as opposed to clinical remission, suggests that monitoring plasmablast levels after treatment might be useful in predicting clinical flares and guiding management accordingly, with prophylactic treatment. Further studies are necessary to evaluate this hypothesis. Broader investigations are also required of the subset of expanded plasmablasts that are $\mathrm{IgG} 4+$.

Expansion in circulating plasmablasts in patients with immune-mediated disease has previously been studied in patients with RA, systemic lupus erythematosus and ulcerative colitis. $^{21} 2627$ 34-36 In RA, elevated levels of circulating plasmablasts, especially elevated levels of late-stage plasmablasts characterised by expression of IgJ mRNA, have been associated with a poor treatment response to peripheral $\mathrm{B}$ cell depletion. ${ }^{35}$ In paediatric and adult patients with ulcerative colitis, those with severe disease have been found to have significantly higher circulating plasmablasts (particularly IgA + plasmablasts) than healthy controls. ${ }^{34} 36$

The observation of elevated plasmablasts in patients with active, untreated IgG4-RD has important implications for understanding the pathophysiology of IgG4-RD as well as the response to treatment. A prior report described oligoclonal B cells in patients with IgG4-RD, ${ }^{37}$ suggesting that circulating plasmablasts may also be oligoclonal (this requires confirmation). Successful treatment of IgG4-RD by the depletion of mature peripheral B cells has been described, but plasmablasts lack surface expression of CD20 and are therefore resistant to direct depletion by anti-CD20 treatment approaches. ${ }^{18}$ The steep decline in plasmablast counts after anti-CD20 treatment likely stems from the depletion of CD20+ precursors. We observed that IgG4-RD patients with elevated plasmablast counts on presentation demonstrate clinical responses to RTX, coincident with a swift decline in both total and IgG4+ plasmablast levels. The utility of total and IgG4+ plasmablast levels as biomarkers of disease activity and predictors of flare requires further investigation.

Our study has both strengths and weaknesses. All patients had IgG4-RD confirmed by biopsy according to recent consensus criteria ${ }^{7}$ and had active disease when their blood samples were evaluated. In addition, none had been treated before study entry. A common challenge in studying patients with inflammatory conditions is the identification of patients before they have received any immunosuppressive therapy. The identification of such patients was a major focus of our study and the main reason why the number of patients studied was relatively small. Although it will be important to confirm and extend these findings in larger studies, our statistical analyses suggest robust differences across many of the comparisons made.

In summary, IgG4-RD is associated with elevated plasmablast counts in the blood. Our results suggest that the plasmablast count is superior to serum IgG4 concentrations as a biomarker for the diagnosis of IgG4-RD. The plasmablast count reflects the extent of disease and may also be useful for the timing of re-treatment.

Funding This study was funded by grants Al 064930 and Al 076505 from the National Institutes of Health and a pilot grant from the Harvard Institute of Translational Immunology supported by the Helmsley Foundation.

Competing interests None.

Ethics approval This study was reviewed and approved by the Massachusetts General Hospital/Partners Institutional Review Board.

Provenance and peer review Not commissioned; externally peer reviewed.

\section{REFERENCES}

1 Stone JH, Zen Y, Deshpande V. IgG4-related disease. N Engl J Med 2012;366:539-51.

2 Carruthers MN, Stone JH, Khosroshahi A. The latest on IgG4-RD: a rapidly emerging disease. Curr Opin Rheumatol 2012;24:60-9.

3 Ebbo M, Daniel L, Pavic M, et al. IgG4-related systemic disease: features and treatment response in a French cohort: results of a multicenter registry. Medicine (Baltimore) 2012;91:49-56.

4 Ferry JA, Deshpande V. IgG4-related disease in the head and neck. Semin Diagn Pathol 2012;29:235-44.

5 Plaza JA, Garrity JA, Dogan A, et al. Orbital inflammation with IgG4-positive plasma cells: manifestation of IgG4 systemic disease. Arch Ophthalmol 2011;129:421-8.

6 Stone JH, Khosroshahi A, Deshpande V, et al. Recommendations for the nomenclature of IgG4-related disease and its individual organ system manifestations. Arthritis Rheum 2012;64:3061-7.

7 Deshpande V, Zen Y, Chan JK, et al. Consensus statement on the pathology of IgG4-related disease. Mod Pathol 2012;25:1181-92.

8 Sato Y, Ohshima K, Ichimura K, et al. Ocular adnexal IgG4-related disease has uniform clinicopathology. Pathol Int 2008;58:465-70.

9 Umehara H, Okazaki K, Masaki Y, et al. A novel clinical entity, IgG4-related disease (IgG4RD): general concept and details. Mod Rheumatol 2012;22:1-14.

10 Shimizu Y, Yamamoto M, Naishiro Y, et al. Necessity of early intervention for IgG4-related disease-delayed treatment induces fibrosis progression. Rheumatology (Oxford) 2013;52:679-83.

11 Brenner I, Roth S, Puppe B, et al. Primary cutaneous marginal zone lymphomas with plasmacytic differentiation show frequent lgG4 expression. Mod Pathol 2013;26:1568-76.

12 Strehl JD, Hartmann A, Agaimy A. Numerous IgG4-positive plasma cells are ubiquitous in diverse localised non-specific chronic inflammatory conditions and need to be distinguished from IgG4-related systemic disorders. J Clin Pathol 2011;64:237-43.

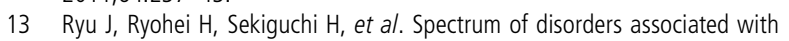
elevated serum IgG4 levels encountered in clinical practice. Int J Rheum Dis 2012:232960.

14 Carruthers M, Khosroshahi A, Augustin T, et al. Diagnostic utility of serum IgG4 concentrations in IgG4-related disease. Ann Rheum Dis 2015;74:14-8.

15 Koike T. IgG4-related disease: why high IgG4 and fibrosis?. Arthritis Res Ther 2013;15:103. 
16 Tsuboi H, Matsuo N, lizuka M, et al. Analysis of lgG4 class switch-related molecules in IgG4-related disease. Arthritis Res Ther 2012;14:R171.

17 Zen Y, Nakanuma Y. Pathogenesis of IgG4-related disease. Curr Opin Rheumatol 2011:23:114-18

18 Khosroshahi A, Carruthers MN, Deshpande V, et al. Rituximab for the treatment of IgG4-related disease: lessons from 10 consecutive patients. Medicine (Baltimore) 2012;91:57-66.

19 Lund FE, Randall TD. Effector and regulatory B cells: modulators of CD4+ T cell immunity. Nat Rev Immunol 2010;10:236-47.

20 Stroopinsky D, Katz T, Rowe JM, et al. Rituximab-induced direct inhibition of T-cell activation. Cancer Immunol Immunother 2012;61:1233-41.

21 Harada $Y$, Kawano MM, Huang $N$, et al. Identification of early plasma cells in peripheral blood and their clinical significance. Br J Haematol 1996;92:184-91.

22 Odendahl M, Jacobi A, Hansen A, et al. Disturbed peripheral B lymphocyte homeostasis in systemic lupus erythematosus. J Immunol 2000;165:5970-9.

23 Fink K. Origin and function of circulating plasmablasts during acute viral infections. Front Immunol 2012;3:1.

24 Jacobi AM, Odendahl M, Reiter K, et al. Correlation between circulating CD27high plasma cells and disease activity in patients with systemic lupus erythematosus. Arthritis Rheum 2003;48:1332-42.

25 Odendahl M, Keitzer R, Wahn U, et al. Perturbations of peripheral B lymphocyte homoeostasis in children with systemic lupus erythematosus. Ann Rheum Dis 2003;62:851-8.

26 Kerkman PF, Rombouts Y, van der Voort El, et al. Circulating plasmablasts/ plasmacells as a source of anticitrullinated protein antibodies in patients with rheumatoid arthritis. Ann Rheum Dis 2013;72:1259-63.

27 Vital EM, Dass S, Buch MH, et al. B cell biomarkers of rituximab responses in systemic lupus erythematosus. Arthritis Rheum 2011;63:3038-47.
28 Iwata S, Saito K, Hirata S, et al. Phenotypic changes of lymphocyte in a patient with lgG4-related disease after corticosteroid therapy. Ann Rheum Dis 2012;71: 2058-9.

29 Carruthers MN, Stone JH, Deshpande V, et al. Development of an IgG4-RD Responder Index. Int J Rheumatol 2012;2012:259408.

30 Hart PA, Topazian MD, Witzig TE, et al. Treatment of relapsing autoimmune pancreatitis with immunomodulators and rituximab: the Mayo Clinic experience. Gut 2013:62:1607-15.

31 Khosroshahi A, Cheryk L, Carruthers M, et al. Spuriously low serum IgG4 concentrations caused by the prozone phenomenon in lgG4-related disease. Arthritis Rheumatol 2014;66:213-17.

32 Khosroshahi A, Stone JH. IgG4-related systemic disease: the age of discovery. Curr Opin Rheumatol 2011;23:72-3.

33 Kamisawa T, Shimosegawa T, Okazaki K, et al. Standard steroid treatment for autoimmune pancreatitis. Gut 2009;58:1504-7.

34 Hosomi S, Oshitani N, Kamata N, et al. Increased numbers of immature plasma cells in peripheral blood specifically overexpress chemokine receptor CXCR3 and CXCR4 in patients with ulcerative colitis. Clin Exp Immunol 2011; 163:215-24

35 Owczarczyk K, Lal P, Abbas AR, et al. A plasmablast biomarker for nonresponse to antibody therapy to CD20 in rheumatoid arthritis. Sci Trans/ Med 2011;3:101ra92.

36 Tarlton NJ, Green CM, Lazarus NH, et al. Plasmablast frequency and trafficking receptor expression are altered in pediatric ulcerative colitis. Inflamm Bowel Dis 2012;18:2381-91.

37 Maillette de Buy Wenniger LJ, Doorenspleet ME, Klarenbeek PL, et al. Immunoglobulin G4+ clones identified by next-generation sequencing dominate the B cell receptor repertoire in immunoglobulin G4 associated cholangitis. Hepatology 2013;57:2390-8 